BMJ Open Sport \& Exercise Medicine

\section{International consensus statement on the psychosocial and policy-related approaches to mental health awareness programmes in sport}

To cite: Breslin G, Smith A, Donohue $B$, et al. International consensus statement on the psychosocial and policy-related approaches to mental health awareness programmes in sport. BMJ Open Sport \& Exercise Medicine 2019;5:e000585. doi:10.1136/ bmjsem-2019-000585

Accepted 1 September 2019
Check for updates

(C) Author(s) (or their employer(s)) 2019. Re-use permitted under CC BY-NC. No commercial re-use. See rights and permissions. Published by BMJ.

For numbered affiliations see end of article.

Correspondence to Dr Gavin Breslin; g.breslin1@ulster.ac.uk

\section{ABSTRACT}

Background Research focused on mental health in sport has revealed a need to develop evidence-supported mental health practices that are sensitive to sport culture, particularly for non-elite athletes. A consensus statement was produced to assist effective mental health awareness in sport and guide programme implementation in this rapidly emerging field.

Method The AGREE Reporting Checklist 2016 was used in two international expert consultation meetings, followed by two online surveys. Experts from 10 countries and over 30 organisations contributed.

Results Six objectives were agreed: (1) to define mental health awareness and service implementation constructs for inclusion in programmes delivered in sporting environments; (2) to identify the need to develop and use valid measures that are developmentally appropriate for use in intervention studies with sporting populations, including measures of mental health that quantify symptom severity but also consider causal and mediating factors that go beyond pathology (ie, well-being and optimisation); (3) to provide guidance on the selection of appropriate models to inform intervention design, implementation and evaluation; (4) to determine minimal competencies of training for those involved in sport to support mental health, those experiencing mental illness and when to refer to mental health professionals; (5) to provide evidence-based guidance for selecting mental health awareness and implementation programmes in sport that acknowledge diversity and are quality assured; and (6) to identify the need for administrators, parents, officials, coaches, athletes and workers to establish important roles in the promotion of mental health in various sports settings.

Conclusion This article presents a consensus statement on recommended psychosocial and policy-related approaches to mental health awareness programmes in sport.

\section{OBJECTIVES}

There has been a rapid expansion of evidence-based public mental health awareness programmes in sport, revealing the need for improvements to mental health programme conception; design and measurement; delivery methods; use of theory; and evaluation approaches in sport. ${ }^{1}$ A review of current policy related to community sport, physical activity and mental health, and illness in England has also concluded that measurable and specific targets that can be used to track positive change related to mental health and illness have not been outlined in much government policy. ${ }^{2}$ Moreover, recently, published consensus statements have focused on elite athletes or elite athlete clinical service provision, ${ }^{3-6}$ overtraining, ${ }^{7}$ or have been specific to a disorder. ${ }^{8}$ Each statement on an individual basis is important, but collectively they do not address mental health awareness, implementation of programmes or evaluation among the wider sporting population beyond elite athletes. For instance, globally, while sports participation rates are high, elite athletes comprise only a very small proportion of total sport participants. ${ }^{9}$ Further, while elite athletes, typically, have access to a range of support systems including medical professionals, sport psychologists and/or professional coaches, such resources are not afforded to subelite or non-elite participants.

Guidelines for the implementation and evaluation of mental health programmes in a non-elite context are crucial because these programmes are also relevant to the vast majority of sport participants worldwide. All participants across the life course of sport would benefit from supporting mental health optimisation. Consensus is therefore required to assist programme designers and deliverers, policymakers and commissioners with respect to: (A) definitions and terminology that can be operationalised when promoting mental health awareness ${ }^{10}$; (B) key design principles including choice of theory, target 
populations, stakeholder involvement and delivery sites; (C) outcomes to measure programme efficacy and effectiveness; and (D) methods for conducting and reporting interventions with sporting population groups (eg, coaches, athletes and officials). Our consensus statement is intended to enhance these areas, and facilitate development of mental health programmes in controlled trials.

A recent Wellbeing in Sport Action Plan 2019-2025 for Northern Ireland ${ }^{11}$ highlighted the importance of sporting environments in improving mental health. Part of this policy action plan suggested the need for consensus in evaluating the evidence for mental health awareness programme implementation at all levels of sport. ${ }^{11}$ Indeed, the Northern Ireland Wellbeing in Sport Action Plan 2019-2025 and the UK Government Mental Health Action Plan for Elite Sport ${ }^{12}$ are the first of their kind internationally to either be supported or developed by governments. Given the current momentum in mental health in sport research, and the increased disclosure by high-profile athletes, it is anticipated that action plans will continue to emerge. We seek to build on previous consensus statements, adding a specific focus on determining effective reporting of mental health awareness and implementation programmes at all levels of sport and for all types of sports workers, and not just programmes which focus on elite athletes. This is necessary to ensure the effective implementation, translation and evaluation of mental health programmes in sport at all levels leading to meaningful benefits at a population level.

\section{STAKEHOLDER INVOLVEMENT}

To assist in carrying out these objectives, the AGREE Reporting Checklist 2016 was adopted. The AGREE Reporting Checklist assists practice guideline developers to improve the completeness and transparency of reporting. The checklist includes six quality domains with 23 key items, providing a systematic process for reporting essential information.

In the development of this international consensus statement, a 2-day consultation meeting of experts was conducted. This included invited international stakeholders representing: research, policy, sporting organisations, practitioners, safeguarding and welfare practitioners, psychiatrists, psychologists and mental health charities. The first event took place at the European College of Sport Science Congress (ECSS) in Dublin, Ireland, on 4 July 2018. ${ }^{13}$ The second took place at the British Psychological Society's (BPS) Division of Sport and Exercise Psychology (DSEP) Annual Conference on 4 December 2018 in Belfast, Northern Ireland. ${ }^{14}$ Both conferences were intentionally targeted as attendees were professionals representing various disciplines from across the field of mental health and sport. Invitations were sent to those who could bring expert knowledge to the discussion and who were not already planning on attending these events.

\section{Consultation 1: ECSS}

Prior to the first meeting, a rationale for the international consensus statement was made available to panel members for their preliminary input. Five presentations were provided by international researchers and policymakers with expertise in mental health and sport. Presentations highlighted: (1) the need for a consensus statement; (2) the available evidence and ongoing research; and (3) policy development needs internationally. Each presentation lasted $20 \mathrm{~min}$, and were as follows: (1) Setting the Context and Rationale for Psychosocial and Policy-Related Approaches to Mental Health and Well-being in Sport-Chartering the Path Towards a Mental Health and Well-being Consensus Statement (GB, Northern Ireland); (2) Tackling the Blues: Designing a Theoretically Informed Sports-Based Mental Health Programme for Children and Young People (AS, England); (3) The Optimum Performance Programme in Sports: A Description of Its Components and Report of Its Randomized Controlled Clinical Trials in Collegiate Athletes (BD, USA); and (4) Integrating Mental Health and Well-being in Sport Into a Government Action Plan: Research to Policy (PD, Northern Ireland). Following the presentations, a discussion was facilitated between the panel and the audience on the content of the presentations and emerging objectives which could be incorporated into an initial draft summary consensus statement.

Four questions were presented: (1) Did delegates acknowledge the need for a consensus statement for psychosocial and policy-related approaches to mental health awareness in sport? (2) Would those involved want to help shape the consensus statement? (3) After reading the draft summary of potential objectives of the statement, what additional information would need to be included, for example, were the aims and methods appropriate? and (4) Were there any additional comments or suggestions? Contributors to the discussion were from eight countries (USA, Taiwan, Italy, Spain, Turkey, England, Northern Ireland and Republic of Ireland).

The summary statement along with a feedback questionnaire was made available online between July and October 2018 to increase the reach of the consultation and to account for the views of those who could not attend the meeting. The survey targeted organisations, policymakers, practitioners and researchers involved in mental health promotion in sport. Responses from the ECSS meeting and the online survey were summarised. Attendees were in broad agreement that an international consensus statement was required and identified seven reasons why (see box 1 ).

In addition to formulating the above rationale, questions were raised about whether the consensus statement should recommend a standardised validated instrument to assess mental health outcomes. The merits of having a single standardised measure were discussed but considered to be too restrictive for both researchers and practitioners. A more robust and inclusive approach 
Box 1 Reasons for the development of an international consensus statement on psychosocial and policy-related approaches to mental health awareness for all involved in sport

Key reasons cited for the development of an international consensus statement

- There has been increasing recognition of mental health issues in sport.

- Growth of sport-based mental health awareness programmes.

- Variation in programme content, design, theory and evaluation.

- Uncertainty about the outcomes or impact of programmes.

- Variations in measurement tools selected—validation and reliability of these tools.

- Lack of agreement on the minimal training requirements for sport and exercise psychologists or mental health club officers in supporting or guiding awareness training programme content and development.

- Necessity for evidence-based recommendations to shape policy.

would be to determine what validated and useful tools are currently available.

It was agreed that the consensus statement should go beyond elite sport and include direction for promoting a positive sport environment, characterised as a climate that encourages mental health help seeking, self-disclosure of mental health-related concerns, stigma reduction specific to mental health and signposting to support those in need of mental health support. It was also acknowledged that promoting mental health and wellbeing in sport requires a shared mandate and is a shared responsibility. ${ }^{15}$ Consequently, there should be a focus on supporting all who participate and work in sport, including employees such as administrators, coaches, sport science support personnel, parents, officials and volunteers.

Findings from the online survey, completed by representatives of 26 organisations from seven countries (England, Northern Ireland, Republic of Ireland, USA, Scotland, Australia and Turkey), including those not at the meeting in July, showed that $100 \%$ of respondents either agreed or strongly agreed that there was a need for an international consensus statement to guide Psychosocial and Policy-Related Approaches to Mental Health Awareness in Sport. From this online survey and discussions at the ECSS meeting, it was confirmed that the consensus statement could inform policymakers and mental health in sport action plans internationally as they emerge.

All respondents indicated they would assist the development of the consensus statement and provided comments on the summary draft. Three key objectives were originally developed: (1) to define mental health awareness constructs for inclusion in programmes in sport; (2) to identify valid measures for use in intervention studies with the sporting population, and establish standards for data collection, analysis and reporting procedures; and (3) to provide policymakers with guidance for implementing awareness programmes in sport. These objectives were subsequently broadened to six, thereby encompassing the opinions of all those involved in the consultation process.

Further views from respondents extended the points addressed above and related to specific interventions, and challenged the importance of the focus of the statement. For instance, one respondent stated: 'My main concern is that the initiative should not omit examination of mental health interventions. Awareness is a big part, but I believe insufficient in making significant change.' This view was widely supported but considered outside the initial remit of the consensus statement on mental health awareness raising. A further comment highlighted the need to engage with those with lived experiences of mental illness. Due to the diversity of opinion expressed in discussions following the initial consultation and online survey it was acknowledged that additional changes to the initial consensus should be considered. This occurred during the second consultation meeting, held at the BPS's DSEP Annual Conference in Belfast, Northern Ireland, in December 2018. This consultation event addressed the feedback from Consultation 1 and involved the suggested inclusion of: (A) those with lived experience of mental illness; and (B) organisations who support those with lived experience of mental illness.

\section{Consultation 2: BPS's DSEP Annual Conference}

Prior to the consultation, the aims of the event were shared with delegates attending the conference via the programme and marketing on the conference website. An information leaflet describing the adapted consensus statement was also produced summarising the revised objectives that emerged from the initial consultation and survey results. At the DSEP conference the second consultation event was scheduled as a panel discussion rather than a traditional keynote address. Seven panel members representing interdisciplinary groups were selected as experts to discuss the six objectives defined in the updated consensus. The panel consisted of the Chair of the BPS DSEP (SC), Royal College of Psychiatrists Special Interest Group in Sport (TR), Clinical Research Trials Researcher (BD), DSEP Training Committee Chair (Dr Ruth Lowry), Childline (Geraldine McConaghy), CEO of Sport Northern Ireland (Antoinette McKeown) and Sociology and Mental Health in Sport Researcher (AS).

The session was independently chaired by Dr John Kremer, a rationale for the session was introduced by GB (Consensus lead) and response data were collected by SS and TJH. The agenda consisted of an update on what had been achieved so far in the development of the consensus statement. ${ }^{14}$ Three questions were posed to the panel and audience: (1) Did the six objectives capture all that is viewed as necessary in the consensus statement? (2) Was each objective fit for purpose? and (3) Were there any other factors that need to be considered? Table 1 outlines 
Table 1 Do the six key objectives capture all that is viewed as necessary in the consensus statement?

\begin{tabular}{|c|c|}
\hline Key objectives & $\begin{array}{l}\text { Responses from the panel, audience and survey that were considered and integrated into the } \\
\text { objectives }\end{array}$ \\
\hline Objective 1 & 'When defining awareness, consider the inclusion of sporting environments.' \\
\hline Objective 2 & $\begin{array}{l}\text { 'Consider having correct measures in place to assess awareness.' } \\
\text { 'The objective is symptom based, what about cause of mental health issues.' } \\
\text { 'Young people should be included in the design of appropriate measures.' } \\
\text { 'Make language of measurements of awareness age appropriate.' }\end{array}$ \\
\hline Objective 3 & 'Consider the inclusion of theory or models for evaluation.' \\
\hline Objective 4 & $\begin{array}{l}\text { 'Coaches should know when to refer to a professional.' } \\
\text { 'Competencies around mental health should be considered in training as a sport and exercise } \\
\text { psychologist.' } \\
\text { 'What is the minimal training required to be a sport and exercise psychologist, this should include mental } \\
\text { health in sport?' } \\
\text { 'If a coach is not trained they need to know who can help.' } \\
\text { 'Quality assuring certification should be considered for awareness training programmes.' } \\
\text { 'Make those involved in sport aware of what follow on care after referral is available.' }\end{array}$ \\
\hline Objective 5 & $\begin{array}{l}\text { 'Public Health Agency quality assurance of mental health awareness trainers.' } \\
\text { 'Training provided in resilience.' } \\
\text { 'Training should be included in a curriculum for athletes, coaches and officials.' }\end{array}$ \\
\hline Objective 6 & $\begin{array}{l}\text { 'Still work to be done here, survey of coaches, directors of governing bodies who look after the people.' } \\
\text { 'Consider peer athlete support.' }\end{array}$ \\
\hline
\end{tabular}

the responses that have been considered after Consultation 2 and integrated into the six original objectives.

Following the event, a second online survey was made available, until 15 February 2019, for those who attended the conference to feed back further recommendations on the content of the revised consensus statement. A key point to emerge from the survey was that the statement should be written using an inclusive non-discriminatory narrative against marginalised individuals, such as those with a disability or in greatest areas of financial need.

On addressing feedback, a draft of the consensus statement was prepared and circulated to all members who expressed an interest in being involved, with an invitation for edits and comments to be returned. All comments received were reviewed and, where applicable, incorporated into revisions of the final consensus statement. Where a suggested change was not incorporated, a written explanation was prepared. After the final iteration, all members of the group were asked to confirm their agreement with the content of the objectives. In total, this international consensus statement evolved from an initial three to six objectives through an 8-month consultation period. The final six consensus statement recommendations are detailed below.

\section{Consensus recommendations}

The six objectives are intended to: (1) inform those developing psychosocial or policy-related mental health awareness programmes; and (2) provide direction for researchers, policymakers and practitioners working in this emerging area (see table 2). As research and practice develops we intend to update the recommendations in the coming decade, and methodologically open the revisions to as wide an audience as possible through online mediums, events and debates.

\section{DISCUSSION}

Recommendations within this consensus statement highlight the need for action towards greater consistency in the development, evaluation and reporting of mental health awareness programmes in sport. First, suggestions were made for appropriately defining mental health awareness and implementation constructs appropriate to sport. We therefore propose that practitioners and policymakers view mental health from a perspective that comprises both mental illness and mental health as two independent, but related, factors through Keyes' two continua model. ${ }^{16}$ Further, we outline a sport-sensitive definition of mental health that participants can relate to. We expanded the WHO's definition, that is; mental health is not merely the absence of illness, but a state of well-being in which those involved in competitive sport realise their purpose and potential, can cope with competitive sport demands and normal life stressors, can work productively and fruitfully, can act autonomously according to their personal values, are able to make a contribution to their community and feel they can seek support when required. As a means of promoting mental health, we suggest that awareness constructs including mental health literacy, ${ }^{17}$ psychological literacy $^{15}$ and mental health self-management ${ }^{18}$ that can be operationalised in the context of interventions should be considered.

Second, we suggest being selective by using psychometrically validated, theory-informed and context-appropriate measurement tools in the evaluation of awareness programmes. ${ }^{19}$ The measures 
Table 2 Finalised international consensus statement recommendations.

Recommendation 1 To define mental health awareness and the key-related constructs for inclusion in programmes delivered in sport environments.

\begin{tabular}{ll} 
Recommendation 2 & $\begin{array}{l}\text { To identify standards for data collection, analysis and reporting procedures, and valid measures } \\
\text { that are age appropriate for use in intervention studies and implementation science with sporting } \\
\text { populations. These may include assessments of mental health that measure symptoms, but also } \\
\text { consider causal factors and mediators, and measures that go beyond pathology (eg, well-being, } \\
\text { resilience, optimisation and team/organisational culture). }\end{array}$ \\
\hline Recommendation 3 & $\begin{array}{l}\text { To provide guidance on the selection of appropriate theories or models and constructs to inform } \\
\text { intervention design, implementation and evaluation. }\end{array}$ \\
\hline Recommendation 4 & $\begin{array}{l}\text { To determine the minimal competencies of mental health awareness training for those involved in } \\
\text { sport to support mental health, those experiencing mental illness and when to refer to professional } \\
\text { services. }\end{array}$ \\
\hline Recommendation 5 & $\begin{array}{l}\text { To provide evidence-based guidance for the selection of mental health awareness and implementation } \\
\text { programmes in sport that acknowledge diversity (eg, age, gender, culture) and are quality assured. }\end{array}$ \\
Recommendation 6 & $\begin{array}{l}\text { To identify what role administrators, parents, officials, coaches, athletes and other workers/volunteers } \\
\text { have in the cocreation of mental health promotion in various sport settings. }\end{array}$ \\
\hline
\end{tabular}

should be aligned with the intended outcomes of the programme; assessing mental health literacy, stigma reduction, help-seeking behaviour, pathological symptoms, and those assessing wellness constructs. Statistical analyses during programme evaluation may consider recommended methodological practices, such as reporting effect sizes, determining adequate sample size and power, and emerging approaches for assessing mediating effects and modelling individual and collective behaviour change over time. ${ }^{20}$ Those involved in programme evaluation could also give consideration to the inclusion of robust longitudinal controlled trial research designs.

Third, programme designers should underpin the intervention components with appropriate theories and models, such that the design, implementation and evaluation of programmes are guided by theoretically derived mechanisms of change through which interventions are more likely to be effective. ${ }^{21} 22$ A number of theoretical models to be considered which to date have shown some success in raising mental health awareness with athletes are: the Theory of Planned Behaviour, Self-Determination Theory, Integrated Behaviour Change Model and Family-Based Behavioural Learning Theory. ${ }^{23}$ The integration of theories has also helped explain additional variance in mental health outcomes ${ }^{18}$ and related health behaviours ${ }^{24}$ and may be considered in the future when developing theory through programme design, implementation and analyses.

Fourth, those involved in the design of programmes should be cognisant of the appropriate level of training that is required for those delivering mental health awareness programmes, ${ }^{25}$ particularly given mental health is a sensitive area. We propose trainers should be aware of sport-specific theories underlying mental health, as Keyes outlines, ${ }^{16}$ including how athletes can function effectively in their sport and experience psychological well-being, while also having mental illness symptoms such as anxiety, depression, bulimia, schizophrenia, and so on. Moreover, trainers require competency in observation skills on how to detect observable changes in moods or behaviours in others; have knowledge of when, where and who to signpost a person if they require help; and finally be able to promote self-care. Adopting a public health approach to programme development, staff training and delivery in and of itself is not enough (ie, using an existing generalist mental health awareness programme), so we suggest that trainers are sensitive to sports cultures and environments and that awareness programmes include sporting case studies as a minimum.

Fifth, those involved in mental health service provision need to consider contextual (eg, culture, family, age, gender) and operational factors (eg, feasibility, length of time, service user experience), without which evidencebased programmes ${ }^{23}$ may not be sustainable or effective. For instance, recent evidence ${ }^{26}$ indicates that collegiate athletes' family problems may predispose them to mental health distress, and therefore serve as a good screening method to assist referrals.

Finally, in recommendation 6 , all of those involved in sport should consider the significant role that all stakeholders (eg, athletes, support staff, national governing bodies, sport associations, media, industry sponsors and clubs) have to play in developing and supporting an open, stigma-free environment in which mental health can be supported within and through sport at all levels. ${ }^{2} 232728$ By implementing these recommendations, it is expected that improved methodological guidance will become available from researchers working alongside stakeholders. Overall, we argue that these approaches will enhance and encourage the creation of valid and reliable evidence base for underpinning future programmes that are designed to increase mental health awareness in sport, and in so doing better inform future psychosocial policy approaches to mental health awareness. 


\section{FUTURE DIRECTIONS}

A rigorous evidence-based implementation of mental health awareness programmes is required across countries to ensure equitable support is available for all involved in sport beyond elite athletes. This international consensus statement provides evidence-based strategies for programme implementation and research to stimulate the development of culturally sensitive mental health promotion efforts that are inclusive of all involved in sport. It is apparent that some plans are already in place, ${ }^{11}{ }^{29}$ while developmental work is currently underway in other countries. Research into what works best in terms of increasing mental health awareness across cultures is also required, and a sharing of this knowledge would be beneficial to the global mental health promotion field. Overcoming the historical personal and social stigma of help seeking requires exploration within sports cultures that have typically lagged behind many other prominent cultural sectors in promoting and addressing mental health. Future work could therefore consider the recommended compatibility and practical guidance that can be provided to national associations on developing a balanced sport performance ethos as well as an open and inclusive environment for mental health help seeking. Finally, there is a relative lack of mental health awareness programmes that address the nuances of competing and working in sport in some countries; in some countries there is a reliance on using a public health model that is not sensitive to sports cultures without adaptation, and in others there is a complete absence of provision. Adopting the above guidance may help inform the future development of policy, practice and science which regards mental health as equally important as sports performance so that all involved in sport are mentally better off.

\section{Author affiliations}

${ }^{1}$ Sports and Exercise Science Research Institute, Ulster University, Jordanstown, UK ${ }^{2}$ Bamford Centre for Mental Health and Wellbeing, Ulster University, Jordanstown, UK

${ }^{3}$ Department of Sport and Physical Activity, Edge Hill University, Ormskirk, UK ${ }^{4}$ Department of Psychology, University of Nevada, Las Vegas, Las Vegas, Nevada, USA

${ }^{5}$ Gaelic Athletic Association, Belfast, Northern Ireland

${ }^{6}$ Faculty of Education, University of Wollongong, Wollongong, New South Wales, Australia

${ }^{7}$ School of Health and Human Sciences, Southern Cross University, Coffs Harbour, New South Wales, Australia

${ }^{8}$ Centre for Athlete Development, Experience \& Performance, Southern Cross

University, Coffs Harbour, New South Wales, Australia

${ }^{9}$ School of Psychology, AECC University College, Bournemouth, UK

${ }^{10} \mathrm{Health}$ Research Institute, University of Limerick, Limerick, Ireland

${ }^{11}$ Cognacity, London, UK

Acknowledgements We acknowledge the contributions of Sport Northern Ireland and Sport and Exercise Science Research Institute for the production of the leaflets at ECSS and BPS Annual Conference. We acknowledge the support provided by Dr John Kremer in chairing the panel discussions and for his edits and comments on an earlier draft of the consensus statement.

Contributors GB coordinated the development of the consensus statement. GB, $\mathrm{AS}, \mathrm{BD}, \mathrm{PD}$ and SC contributed to the development and delivery of presentations at the consultation meetings. GB, TJH and SS analysed the responses from the panel feedback and two surveys. GB wrote the first draft of the article. All other authors contributed to subsequent drafts and the final version after peer review.

Funding The authors have not declared a specific grant for this research from any funding agency in the public, commercial or not-for-profit sectors.

Competing interests None declared.

Patient consent for publication Not required.

Ethics approval Ethical approval was granted by the Ulster University Research Ethics Committee.

Provenance and peer review Not commissioned; externally peer reviewed.

Data availability statement Data are available upon reasonable request.

Open access This is an open access article distributed in accordance with the Creative Commons Attribution Non Commercial (CC BY-NC 4.0) license, which permits others to distribute, remix, adapt, build upon this work non-commercially, and license their derivative works on different terms, provided the original work is properly cited, appropriate credit is given, any changes made indicated, and the use is non-commercial. See: http://creativecommons.org/licenses/by-nc/4.0/.

\section{REFERENCES}

1. Breslin G, Shannon S, Haughey T, et al. A systematic review of interventions to increase awareness of mental health and well-being in athletes, coaches and officials. Syst Rev 2017;6:1-15.

2. Smith $A$, Jones J, Houghton L, et al. A political spectator sport or policy priority? A review of sport, physical activity and public mental health policy. International Journal of Sport Policy and Politics 2016:8:593-607.

3. Schinke RJ, Stambulova NB, Si G, et al. International society of sport psychology position stand: Athletes' mental health, performance, and development. Int J Sport Exerc Psychol 2017.

4. Henriksen K, Schinke R, Moesch K, et al. Consensus statement on improving the mental health of high performance athletes. International Journal of Sport and Exercise Psychology 2019;14:1-8.

5. Moesch K, Kenttä G, Kleinert J, et al. FEPSAC position statement: mental health disorders in elite athletes and models of service provision. Psychol Sport Exerc 2018;38:61-71.

6. Schinke RJ, Stambulova NB, Si G, et al. International society of sport psychology position stand: Athletes' mental health, performance, and development. Int J Sport Exerc Psychol 2018;16:622-39.

7. Meeusen R, Duclos M, Foster C, et al. Prevention, diagnosis and treatment of the overtraining syndrome: joint consensus statement of the European College of sport science (ECSS) and the American College of sports medicine (ACSM). Eur J Sport Sci 2013;13:1-24.

8. Mountjoy M, Sundgot-Borgen J, Burke L, et al. The IOC consensus statement: beyond the female athlete Triad-Relative energy deficiency in sport (RED-S). Br J Sports Med 2014;48:491-7.

9. Aubert S, Barnes JD, Abdeta C, et al. Global matrix 3.0 physical activity report card grades for children and youth: results and analysis from 49 countries. $J$ Phys Act Health 2018;15:S251-73.

10. Lundqvist $\mathrm{C}$. Well-Being in competitive sports - The feel-good factor? A review of conceptual considerations of well-being. Int Rev Sport Exerc Psychol 2011;4:109-27.

11. Donnelly P, Breslin G, 2018. Available: https://www.communitiesni.gov.uk/publications/wellbeing-sport-action-plan-2018-2023 [Accessed 16 Oct 2018].

12. Department for Digital,. Culture, Media \& Sport (2018) Mental Health and Elite Sport Action Plan. Available: https://www.gov.uk/ government/publications/mental-health-and-elite-sport-action-plan [Accessed 22 Feb 19].

13. Breslin G. Psychosocial and policy related approaches to mental health and wellbeing in Sport- Chartering the path towards a mental health and wellbeing consensus statement. European College sports science, Dublin, page 4, 2018. Available: http://ecss-congress.eu/ 2018/assets/images/DublinAnnouncement.pdf [Accessed 24th Oct 2018].

14. Breslin G. Psychosocial and Policy-Related approaches to mental health awareness in sport: developing a consensus statement. British psychological Society, division of sport and exercise psychology annual conference, Belfast. page 8, 2018. Available: https://www.bps.org.uk/sites/bps.org.uk/files/Events\%20-\%20Files/ DSEP2018\%20Draft\%20Programme\%20V12.pdf [Accessed 24th Oct 2018].

15. MacIntyre TE, Jones M, Brewer BW, et al. Editorial: mental health challenges in elite sport: balancing risk with reward. Front Psychol 2017;8:1892. 
16. Westerhof GJ, Keyes CLM. Mental illness and mental health: the two continua model across the lifespan. J Adult Dev 2010;17:110-9.

17. Jorm AF. Mental health literacy. Public knowledge and beliefs about mental disorders. Br J Psychiatry 2000;177:396-401.

18. Shannon S, Breslin G, Haughey T, et al. Predicting Student-Athlete and Non-Athletes' Intentions to Self-Manage Mental Health: Testing an Integrated Behaviour Change Model. Ment Health Prev 2019;13:92-9.

19. Hagger MS, Chatzisarantis NLD. Assumptions in research in sport and exercise psychology. Psychol Sport Exerc 2009;10:511-9.

20. Myers ND, Ntoumanis N, Gunnell KE, et al. A review of some emergent quantitative analyses in sport and exercise psychology. Int Rev Sport Exerc Psychol 2018;11:70-100.

21. Jones J, O'Keeffe H, Smith A. Tackling the Blues: a sport and education based mental health programme for children and young people. In: Breslin G, Leavey G, eds. Mental health and well-being interventions in sport: a case study analysis. London: Routledge, 2019.

22. Davis R, Campbell R, Hildon Z, et al. Theories of behaviour and behaviour change across the social and behavioural sciences: a scoping review. Health Psychol Rev 2015;9:323-44.
23. Breslin G, Leavey G. Mental health and well-being interventions in sport: research, theory and practice. London, England: Routledge, 2019.

24. Hagger MS. Theoretical integration in health psychology: unifying ideas and complementary explanations. Br J Health Psychol 2009;14:189-94.

25. Proctor EK, Landsverk J, Aarons G, et al. Implementation research in mental health services: an emerging science with conceptual, methodological, and training challenges. Adm Policy Ment Health 2009;36:24-34.

26. Hussey JE, Donohue B, Barchard KA, et al. Family contributions to sport performance and their utility in predicting appropriate referrals to mental health optimization programmes. Eur J Sport Sci 2019;19:972-82.

27. RoderickM, SmithA, PotracP. The sociology of sports work, emotions and mental health: Scoping the field and future directions. Sociol Sport J2017;34:99-107.

28. Atkinson M. Sport, mental illness and sociology. Oxford: Elsevier, 2019.

29. Breslin G, Donnelly P, Shannon S, et al. Four steps in the development of a wellbeing in sport action plan for Northern Ireland: research to practice. Munster Germany: FEPSAC, 2019. 